\title{
Endoscopic eyebrow-eyelid sparing orbital exentration in mucormycosis
}

\author{
Manish Munjal ${ }^{1 *}$, Gagandeep Singh ${ }^{2 *}$, Porshia Rishi1 ${ }^{1}$, Harjinder Singh ${ }^{1}$, Shubam Munjal ${ }^{1}$, \\ Shivam Talwar', Salony Sharma ${ }^{1}$, Tej Mehar Chugh ${ }^{3}$
}

${ }^{1}$ Department of Otorhinolaryngology, ${ }^{2}$ Department of Neurology, ${ }^{3}$ Intern, Dayanand Medical College and Hospital, Ludhiana, Punjab, India

Received: 31 December 2020

Revised: 11 February 2021

Accepted: 12 February 2021

\author{
*Correspondence: \\ Dr. Manish Munjal, \\ E-mail: manishmunjal30@gmail.com
}

Copyright: (C) the author(s), publisher and licensee Medip Academy. This is an open-access article distributed under the terms of the Creative Commons Attribution Non-Commercial License, which permits unrestricted non-commercial use, distribution, and reproduction in any medium, provided the original work is properly cited.

\begin{abstract}
The intra-vascular invasion progressively halts the blood flow in the arterial distributaries and the venous tributaries and finally a "blue black", wet gangrenous region is noted. Later the underlying bone too becomes fragile and crumbles off. Removal of necrotic debris remains the main stay of management. Removal of foci of infection, the orbital soft tissues utilizing high resolution endoscopic modality is elaborated upon.
\end{abstract}

Keywords: Endoscopic, Exenteration, Mucormycosis, High resolution, Orbital

\section{INTRODUCTION}

Sino-nasal fungal infestation by the Rhizopus species, namely Mucormycosis in the immunocompromised diabetics usually has an aggressive spread. ${ }^{1}$ The mucor invades the venous and arterial arcades in the nose. Thence it traverses inside the blood vessels to the adjacent regions. The hard palate inferiorly, the orbits, pterygopalatine, and the infra temporal fossa laterally and the frontal lobe superiorly. The orbital manifestation is usually a sudden loss of vision due to occlusion of the central retinal artery or the ophthalmic artery. The anterior and posterior ethmoidal vessels are the suggested channels of intraconal spread in subjects with an intact lamina papyracea. The other orbital manifestation being axial proptosis with an accompanying thrombosis in the superior and inferior ophthalmic veins. Imaging studies, computed tomography and magnetic resonance imaging usually clinch the diagnosis and even dictate the limits of debridement. Septo-turbinal radical nasal intervention is the preferred therapeutic modality with reluctance on mentioning the orbital intervention. We hereby present an intriguing case report in which endoscopic orbital exenteration was performed after 10 days of the endoscopic nasal debridement.

\section{CASE REPORT}

62 year old male presented to the ear nose and throat outpatient department (ENT OPD) with complaint of right a sided ptosis and sudden vision loss for two days. Patient was known case of uncontrolled diabetes mellitus on irregular treatment. On examination his perception of light in the right eye was negative and direct pupillary reaction was absent with restriction of extra ocular movements in all quadrants resulting in total ophthalmoplegia. Left eye was subjectively normal. On fundus examination right eye showed proliferative diabetic retinopathy with central retinal artery occlusion with optic atrophy. Guarded visual prognosis was explained to the patient. He was advised orbital exenteration along with sinonasal debridement.

Magnetic resonance imaging brain and orbit showed (Figure 2 and 3) enhancing T2 hyperintense mucosal hypertrophy along the walls of maxillary, sphenoid, ethmoid and right frontal sinuses. Enhancing soft tissue is seen along the optic nerve on right side and the orbital apex. Preseptal edema was seen on the right side with no evidence of proptosis. Globes were symmetrical. Extraoccular muscles were symmetrical in size but 
muscles on right side showed mild hyperintensity. Suggestive of orbital cellulitis and fungal cerebritis involving basifrontal regions. Patient underwent endoscopic nasal debridement in which inferior turbinate, middle turbinate, posterior septum and mucosa over cribriform area was found necrosed and was removed. At that time due to emotional issue, he was not consenting for orbital exenteration. Patient was put on intravenous (i/v) liposomal amphotericin B, and administered a cumulative dose of $5500 \mathrm{mg}$.

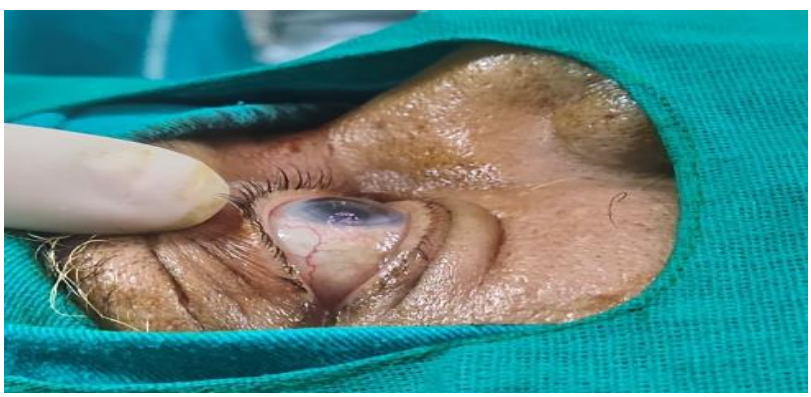

Figure 1: Patient with frozen eye (total ophthalmoplegia) right eye.

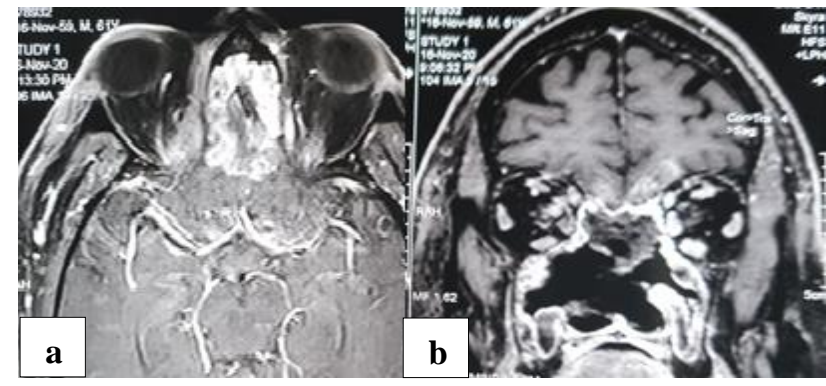

Figure 2: MRI (a) axial section showing opacity at medial side orbital apex right eye compared to the left and (b) coronal section prominent superior ophthalmic vein (orange arrow) right side.

Patient was taken up for endoscopic assisted eyelid sparing orbital exenteration after a period of one month when there was no improvement with medical management. Palpebral orbital elliptical incision was marked (Figure 3B) just above and below the eyelashes after performing 3 suture tarsoraphy (Figure 3A).

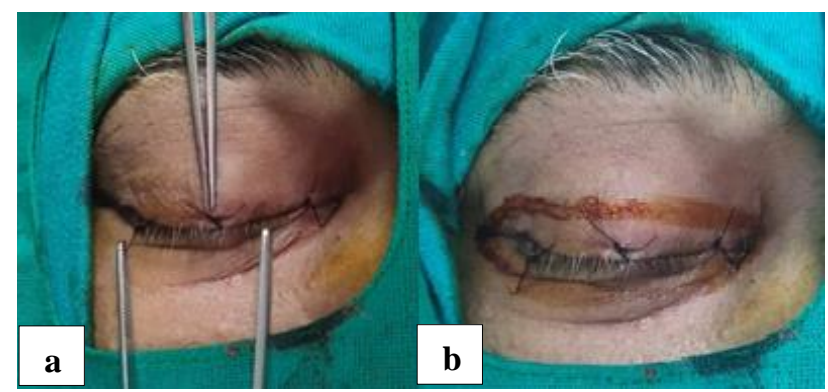

Figure 3: (a) Three stitch tarsorraphy and (b) elliptical marking of eye lid sparing incision.
The skin was elevated from the tarsal plates with monopolar cautery up to the orbital rims circumferentially (Figure 4). Periosteum was then incised circumferentially medial to the orbital rim, exposing the underlying bone. The orbital contents were mobilized in the relatively avascular subperiosteal plane inferiorly, laterally and superiorly. Lateral orbital extraconal dissection showed the zygomatic vessels (blue arrow) and contents of superior orbital fissure which were transected (Figure 5).

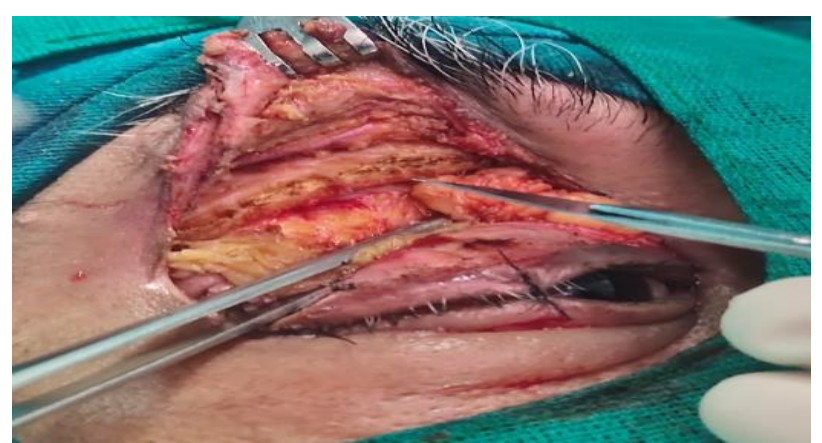

Figure 4: Skin elevation till orbital rims (orange arrow).

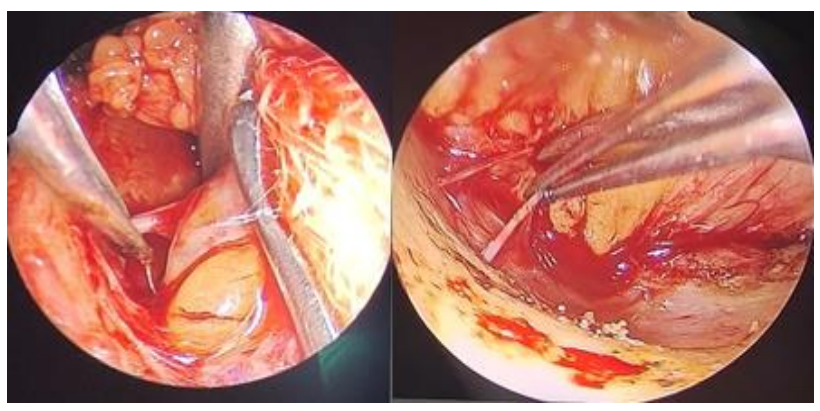

Figure 5: Lateral orbital extraconal dissection showing zygomatic vessels (blue arrow) and contents of superior orbital fissure.

After inferior mobilization infra orbital artery, vein and nerve were bipolarized (Figure 6). Anterior, posterior ethmoidal vessels, nerves and optic nerve were identified during medial mobilization along the frontoethmoidal suture and were transected and cauterized (Figure 9).

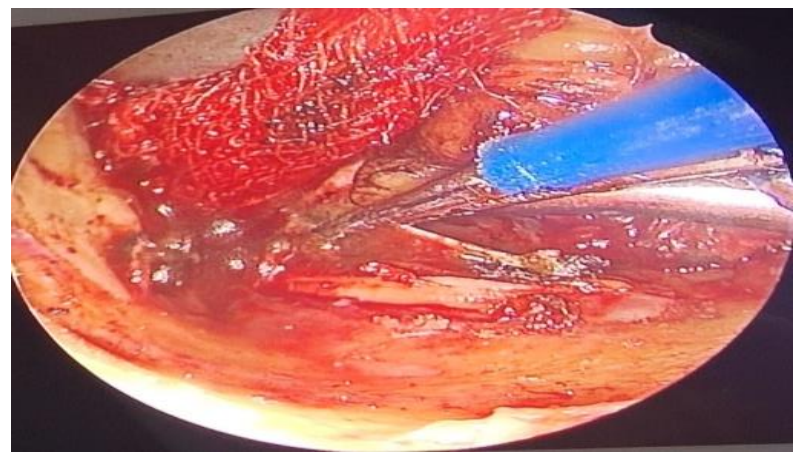

Figure 6: Inferior mobilization to show infra orbital artery vein and nerve (green arrow). 


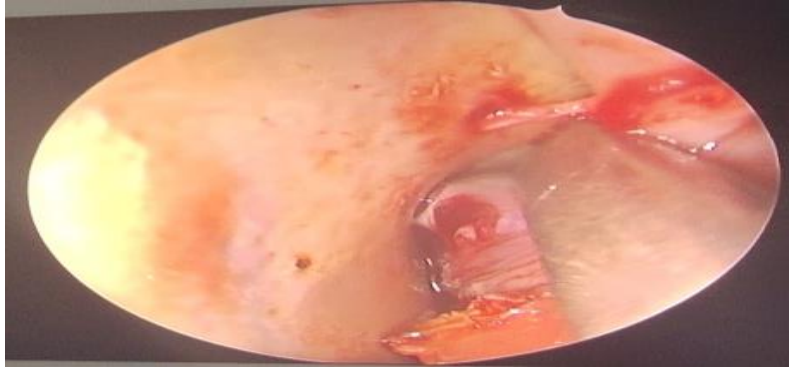

Figure 7: Medial mobilization to show anterior, posterior ethmoidal vessels, (black arrow) nerves and optic nerve (blue arrow).

The ocular muscles and optic nerve were transected after bipolarizing the ophthalmic vessels at the orbital apex. The superior orbital fissure, inferior orbital fissure and optic foramen were identified at the apex of the orbital socket after delivering the orbital contents (Figure 8). The upper and lower eyelid skin was sutured lining the orbital socket (Figure 9).

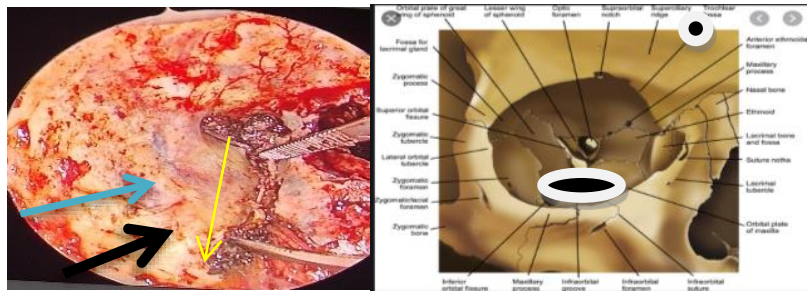

Figure 8: Endoscopic view of the orbital socket showing superior orbital fissure (blue arrow), inferior orbital fissure (black arrow) and optic foramen (yellow) and schematic picture showing the orbital apex right side.

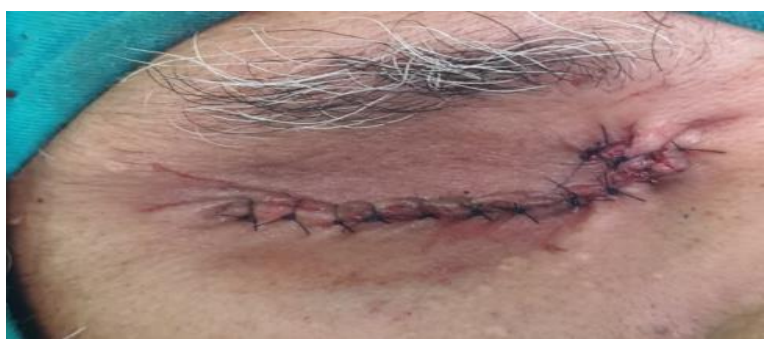

Figure 9: The inverted skin of eyelids, lining the orbit socket.

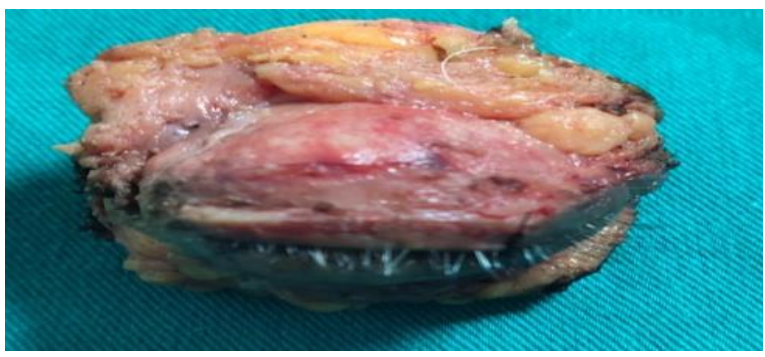

Figure 10: The conical resected specimen within the periosteum.

\section{DISCUSSION}

Mucormycosis is a rapidly progressive, fatal fungal infection caused by filamentous fungi of the Mucoraceae family. The predominant species of mucormycosis include Rhizopus and mucor. ${ }^{1}$ Uncontrolled diabetes mellitus (DM), immunosuppressive medications, steroids, neutropenia, acquired immune deficiency syndrome, dialysis patients on deferoxamine, malnutrition and hematologic malignancies are the common predisposing factors for mucormycosis. ${ }^{2}$

Our patient too was an uncontrolled diabetic for last 10 years. A risk factor for mucormycosis.

The route of spread of airborne spores of the fungi is through inhalation, or through a wound, or an extraction socket. The spores gain access to the nasal or oral cavity and then invade blood vessels to spread to the orbit and the brain. $^{3}$

Orbital exenteration is the surgical removal of the tooth orbital contents, including the eyeball. Exenteration may be subtotal, total, extended exenteration, or radical exenteration, depending on the amount of orbital contents and its periphery, excised.

Meyer and Zaoli's classified orbital exenteration in 1970 as: type I - palpebral skin and conjunctiva are spared. Type II - only the palpebral skin is spared and the eyeball and its appendages are removed with the conjunctiva, type III both eyelids are removed with orbital contents, and type IV - the eyeball, eyelids and appendages of the eye are removed with the involved bony structures. ${ }^{4,5}$

Yeatts divided orbital exenteration into two categories: total exenteration as removal of the entire orbital contents with or without sacrifice of skin of eyelid, and subtotal exenteration as partial removal of orbital tissues with sacrifice of the eye, which can be considered as an extended enucleation. ${ }^{6}$

Exenteration can be performed by either an eyelidsacrificing or eyelid-sparing technique.

The orbital cavity may be managed in a number of ways after exenteration. Patients may be left with the orbital cavity exposed with or without an ocular prosthesis. When wearing prosthesis is not an option, patients prefer to have the cavity filled with a flap.

An eyelid-sparing technique popularized by Coston and Small in 2000, is a modification of the total exenteration technique, which spares parts of both the eyelids with transverse blepharorrhaphy to cover the orbit. ${ }^{7}$

In our patient eyelid sparing endoscopic assisted orbital exenteration was undertaken to meticulously dissect out the neurovascular components of the orbital apex and the frontoethmoid canals under magnified visualization on a 
high resolution monitor .There by there was minimal bleed on transecting the optic nerve and inferior ophthalmic vessels. Moreover an eyelid sparing technique ensured better cosmesis and early rehabilitation as the eyelids were sutured together at completion of the surgery by making palpebral incisions just above and below the eyelashes.

The favorable outcome was attributed to rapid correction of the underlying medical condition; wide local excision and debridement of all involved and devitalized sinonasal and periorbital tissue for the "reach" of antifungals to work.

Endoscopic power-assisted orbital exenteration is advantageous as there is direct transnasal control of the ophthalmic artery as it emerges from the optic foramen and the ability to preserve the uninvolved superior and lateral periorbita. This facilitates mucosal coverage of the exenterated space and obviates the need for free skin or tissue grafts .Blood loss and operative time are minimized with wide endoscopic view. ${ }^{8}$

Emerging complications such as postoperative orbital bone exposure, osteoradionecrosis, extra-intracranial fistulas and cerebral abscesses need to be taken into account when choosing the mode of reconstruction.

Osseo integrated implants, split thickness skin graft, transcutaneous abutments, oculoplastic prosthesis, pedicled and free flaps are the various modes of reconstruction used after orbital exenteration. ${ }^{9}$

The large numbers of flaps have been described in literature to cover the bare orbital bones - the temporalis muscle pedicled flap, the galea fascia or pericranial flap, the myocutaneous pedicled flap and the revascularized free flap. ${ }^{10,11}$

\section{CONCLUSION}

Endoscopic assisted orbital eye lid sparing orbital exenteration is an excellent modality with high resolution visualization, precise dissection, minimum blood loss, early healing, with acceptable cosmesis and early prosthetic rehabilitation.

\section{Funding: No funding sources}

Conflict of interest: None declared

Ethical approval: Not required

\section{REFERENCES}

1. Viterbo S, Fasolis M, Garzino-Demo P, Griffa A, Boffano $\mathrm{P}$, Iaquinta $\mathrm{C}$, et al. Management and outcomes of three cases of rhino-cerebral mucormycosis. Oral Surg. 2011;112:69-74.

2. Kim J, Fortson JK, Cook HE. A fatal outcome from rhino-cerebral mucormycosis after dental extractions: a case report. J Oral Maxillofac Surg. 2001;59:693-7.

3. Pinto ME, Manrique HA, Guevara X, Acosta M, Villena JE, Solis J. Hyperglycemic hyperosmolar state and rhino-orbital mucormycosis. Diabetes Res Clin Pract. 2011;91:37-9.

4. Zaoli G, Motta G. La chirurgiaricostruttivanelcancrodellatesta e del collo. Padova: PiccinEditore. 1978.

5. Radici M, Bicciolo G, Palma O, Bozza F. Il massicciofacciale. In: De Campora E, Marzetti F, editors. La chirurgiaoncologicadellatesta e del collo. Pisa: PaciniEditore. 1996;345-81.

6. Yeatts RP. The esthetics of orbital exenteration. Am J Ophthalmol. 2005;139:152-3.

7. Honavar SG, Rao R. Enucleation and Exenteration. In Surgical Ophthalmic Oncology. Springer, Cham. 2019;131-9.

8. Batra PS, Lanza DC. Endoscopic power-assisted orbital exenteration. Am J Rhinol. 2005;19(3):297301.

9. Chepeha DB, Moyer JS, Bradford CR, Prince ME, Marentette L, Teknos TN. Osseocutaneous radial forearm free tissue transfer for repair of complex midfacial defects. Arch Otolaryngol Head Neck Surg. 2005;131:513-7.

10. Ariyan S. The pectoralis major myocutaneous flap. A versatile flap for reconstruction in the head and neck. Plast Reconstr Surg. 1979;63:73-81.

11. Cameron M, Gilbert PM, Mulhern MG, Sneddon KJ. Synchronous reconstruction of the exenterated orbit with a pericranial flap, skin graft and osseointegrated implants. Orbit. 2005;24:153-8.

Cite this article as: Munjal M, Singh G, Rishi $P$, Singh H, Munjal S, Talwar S, et al. Endoscopic eyebrow-eyelid sparing orbital exentration in mucormycosis. Int J Otorhinolaryngol Head Neck Surg 2021;7:551-4. 